\title{
Habilidades investigativas -en relación al uso de las TIC- a desarrollar en estudiantes de carreras con perfil informático.
}

\section{Research skills -in relation to the use of ICT- students develop careers in computer profile.}

\author{
Odiel Estrada Molina \\ oestrada@uci.cu \\ Universidad de las Ciencias Informáticas
}

\begin{abstract}
Resumen:
En investigaciones educativas, se argumentan potencialidades que ofrecen las tecnologías de la información y las comunicaciones (TIC) y especialmente las redes sociales y académicas en el desarrollo de habilidades investigativas del estudiantado. Se realizó un análisis bibliográfico evidenciándose que no se han determinado cuáles de estas habilidades pero asociadas al empleo de las TIC, deben desarrollarse en el estudiantado según las exigencias curriculares de las carreras con perfil informático. Partiendo de estas deficiencias, se determinó como problema de investigación ¿Qué habilidades investigativas asociadas al empleo de las TIC deben de desarrollarse en el estudiantado de carreras con perfil informático? El objeto de la investigación es desarrollo de habilidades investigativas en carreras con perfil informático; el campo de acción: habilidades investigativas asociadas al empleo de las TIC. El objetivo es determinar habilidades investigativas a desarrollar en los estudiantes de carreras con perfil informático en correspondencia al empleo de las TIC. Se obtuvo como resultado un conjunto de habilidades investigativas asociadas al empleo de las TIC y sus respectivos indicadores, que deben dominar los estudiantes de carreras con perfil informático.
\end{abstract}

Palabas Clave: estudiantes, desarrollo, ingeniería informática, habilidades investigativas, tecnología de la información y las comunicaciones.

\begin{abstract}
:
In educational research, it is argued potential offered by information and communications technology (ICT) and especially social and academic networks in developing research skills of students. A literature review was performed, demonstrating that they have not determined which of these skills (associated with the use of ICT), to be developed in the students according to the curriculum requirements of computer engineering. Therefore, it was determined as a research problem: what research skills associated with the use of ICT should be developed in the students of computer engineering or similar profiles? The object of the research is to develop research skills in computer engineering; the field of action: research skills associated with the use of ICT. The objective is to determine research skills associated with the use of ICT should be developed in the students of computer engineering or similar profiles. In this paper presents a set of research skills associated with the use of ICT and their respective indicators.
\end{abstract}

Keywords: students, development, computer engineering, research skills, information and communications technology. 


\section{Introducción}

La formación investigativa del alumno consiste en la preparación en los aspectos cognoscitivos y afectivos del trabajo científico y está determinada por tres aspectos fundamentales:

1. Conocimientos y habilidades de la ciencia particular, necesarios para el desempeño profesional en aras de satisfacer la demanda social.

2. Conocimientos y habilidades generales del trabajo científico necesarios para el desarrollo eficaz de investigaciones científicas.

3. Cualidades de la personalidad que deben caracterizar al investigador científico en su sociedad, o sea, actitud científica.

En este sentido la formación investigativa de los estudiantes no se limita a la adquisición de conocimientos teóricos acerca de los métodos, estrategias y las técnicas de la investigación científica, sino que también contempla la formación de una actitud verdaderamente científica que les permita actuar de forma efectiva en la solución de sus tareas. Las habilidades investigativas permiten la ejecución de los conocimientos teóricos acerca de la metodología de la investigación científica en la práctica investigativas, utilizando para ello técnicas, métodos, estrategias y metodologías para la realización de una investigación. La actividad investigativa si bien no solo está conformada por las habilidades investigativas, contiene a su vez los componentes actitudinales y motivacionales que en su integración contribuyen a la realización exitosa de la investigación.

En el caso de las habilidades investigativas, esta le permite al sujeto: 1) la vía de recopilar, seleccionar, clasificar, manipular, asimilar y transformar la información existente asociada a la investigación que esté realizando, 2) la realización de una investigación a partir de las etapas lógicas de la investigación científica; 3) la superación profesional del sujeto; 4) la aplicación y desarrollo de la ciencia; 5) la socialización de los resultados obtenidos con la comunidad científica: y 6) la respuesta a una necesidad determinada.

Desde hace más de una década se ha venido potenciado el uso de las Tecnologías de la Información y las Comunicaciones (TIC) en el ámbito educativo, como es en el caso de la Educación Superior. Su empleo ha permitido, entre otras potencialidades, que el proceso de enseñanza-aprendizaje se diversifique hacia la educación semi-presencial y a distancia, y hacia la toma de decisiones asociadas al uso de métodos de inteligencia artificial y estadísticos para evaluar el nivel de aprendizaje de los estudiantes.

Para la realización de este trabajo se asume como concepto de TIC, el expresado por (Cobo, 2009) cuando afirma: "Las TIC son dispositivos tecnológicos (hardware y software) que permiten editar, producir, almacenar, intercambiar y transmitir datos entre diferentes sistemas de información que cuentan con protocolos comunes. Estas aplicaciones, que integran medios de informática, telecomunicaciones y redes, posibilitan tanto la comunicación y colaboración interpersonal (persona a persona) como la multidireccional (uno a muchos o muchos a muchos). Estas herramientas desempeñan un papel sustantivo en la generación, intercambio, difusión, gestión y acceso al conocimiento.” (p. 18). 
La necesidad de las TIC en las instituciones educativas ha sido expresadas por varios autores en los últimos 13 años como (Bosco, 2005), (British Educational Communications and Technology Agency (BECTA), 2007) y la (European Commision, 2006) todos estos autores e instituciones coinciden que el uso de las TIC ha permitido entre otros aspectos, crear un plan institucional de docencia virtual, el empleo de plataformas de software de uso educativo, así como el desarrollo de habilidades.

La formación y desarrollo de habilidades investigativas asociadas al uso de las TIC han sido abordado por varios autores como (Velásquez, 2007), (Barnett, 2008), (Castillo, 2008, 2011), (Balbo, 2008), (Chu, Chow, Tse, y Kuhlthau, 2008), donde se confirma que debido a las exigencias educativas, científicas y tecnológicas del Siglo XXI, se pueden contribuir de manera más eficiente al desarrollo de dichas habilidades.

En la bibliografía consultada se refleja que no se han determinado que habilidades investigativas -en relación al uso de las TIC- se pueden desarrollar en correspondencia a las etapas de la investigación científica ya que solo se enmarcan principalmente en herramientas y tecnologías para la gestión bibliográfica, la redacción de informes, la exposición oral de los resultados, entre otros argumentados en la sección de materiales y métodos. Tampoco se abordan que habilidades investigativas se debe desarrollar según exigencias curriculares de las carreras con perfil informático.

En el caso de las carreras con perfil informático, dígase Ingeniería en Sistemas Informáticos, Licenciatura en Ciencias de la Computación e Ingeniería en Ciencias Informáticas, entre otras, sus egresados deben desarrollar habilidades investigativas en varias esferas de las Ciencias Computacionales y de la Ingeniería de Software, y una de estas es el Proceso de Desarrollo de Software (PDS). Si bien las habilidades investigativas en su conceptualización son aplicables a cualquier actividad investigativa, en el caso de las carreras con perfil informático su operacionalización está sujeta a ciertas elementos distintivos acordes al PDS, por lo que su descripción ayudaría a su desarrollo por parte de los profesionales que están a cargo de la formación y desarrollo de habilidades investigativas en los estudiantes de pregrado.

Según las tendencias en la formación del ingeniero informático a nivel internacional, el CAREER SPACE (consorcio conformado por las grandes compañías de las Tecnologías de la Información y las Comunicaciones), la ACM-IEEE (Asociación de Máquinas Computadoras y Instituto de Ingenieros Eléctricos y Electrónicos), el Espacio Europeo de Educación Superior y ABET (acreditación de programas de educación universitaria o terciaria en disciplinas de ciencias aplicadas, ciencias de la computación, ingeniería y tecnología) afirman la necesidad del desarrollo de las habilidades investigativas en este tipo de carreras, y como particularidad deben estar dirigidas principalmente desde el PDS.

El objetivo de este trabajo es determinar habilidades investigativas -en relación al uso de las TIC- en correspondencia a las carreras con perfil informático.

El trabajo posee la siguiente estructura: Introducción, en la que se establecen la necesidad de la investiagción así como el objetivo a cumplir; Materiales y métodos, en el que se abordan los procedimientos empleados así como los métodos cientificios; Resultados, en el que se describen los resultados obtenidos; Discusión, en la que se discute los resultados y por último las conclusiones.

\section{Materiales y métodos}

Se realizó un análisis bibliográfico a más de 40 investigaciones asociadas a las habilidades investigativas y también a competencias investigativas pues si bien son términos diferentes, la 
competencia integra conocimientos, habilidades, actividades, motivaciones, entre otros elementos de la personalidad.

Entre las principales investigaciones asociadas a estas fin se encuentra (Quigley y Kuhne, 1996), (Rodríguez, 2000), (Irigoyen y Vargas, 2002), (Pla, 2005), (Delamare Le Deist y Winterton, 2005), (Burke, Schlenk, Sereika, Cohen, Happ y Dorman, 2005), (Arbeláez, Orlando, y Ilce, 2006), (Muñoz, Quintero, y Munévar, 2006), (Facundo, Patricia, Carlos, Ruíz,Claro,Vanegas, 2007), (Maldonado, Landazábal, y Hernández, 2007), (Velásquez, 2007), (Barnett, 2008), (Castillo, 2008, 2011), (Balbo, 2008), (Chu, Chow, Tse, y Kuhlthau, 2008), (Tobón, 2008, Tobón, 2008a), (Gayol, Montenegro, Tarrés, y D’Ottavio, 2008), (Contreras, 2010), (Ortega y Jaik, 2010), (Hsing-fen, Miozzoa, y Laredob, 2010), (Bolgzda y Olehnovica, 2012), (Association pour l'emploi des cadres (France), Deloitte (France), 2010), (Baltrūna, 2013) y (Bezrukova y Bezrukov, 2013).

En el análisis realizado y a partir de la sistematización teórica publicada anteriormente en (Estrada, 2014), se refleja que no se han determinado que habilidades investigativas -en relación al uso de las TIC- se pueden desarrollar en el estudiantado en correspondencia a las etapas de la investigación científica ya que solo se enmarcan principalmente en herramientas y tecnologías para la gestión bibliográfica, la redacción de informes, la exposición oral de los resultados, el uso de la estadística, la búsqueda y procedimiento de la información científica en la Web y el intercambio en redes y comunidades profesionales y académicas. Tampoco se abordan que habilidades investigativas -en relación al uso de las TIC- se debe desarrollar según exigencias curriculares de las carreras con perfil informático.

\section{Resultados}

Diversos autores asumen diversas etapas secuenciales de la investigación científica, pero tomando en consideración a (Fernández, Llivina, Arencibia, Hernández, y Castellanos, 2003), estas pueden ser: 1) la exploración de la realidad; 2) la planificación de la actividad científica; 3) la ejecución de la investigación; 4) el procesamiento de la información; 5) la comunicación de los resultados científicotécnico; y 6) la introducción y generalización de dichos resultados.

En la etapa de "exploración de la realidad", el investigador debe: elaborar de un marco teóricoreferencial acerca del objeto de estudio; identificar la situación problémica existente en una esfera concreta de la realidad; y determinar el problema científico de la investigación.

En esta etapa el investigador debe evidenciar el uso de las TIC, desde lo cual ha de demostrar el dominio ciertas habilidades, en dependencia de las condiciones tencológicas de la institución, el tipo de investigación a realizar y los conocimientos asociados a las tecnologías que posea.

Las habilidades asociadas al uso de lsa TIC para la primera estapa son:

$>$ Elaborar encuestas y entrevistas con la utilización de las TIC. Esta habilidad le permitirá al investigador recoger datos empíricos desde diferentes lugares de la institución sin necesidad de utilizar de forma presencial estas técnicas de obtención de información. Sus indicadores pudieran ser: 1) Selección efectiva de las herramientas informáticas a utilizar para la confección de encuestas y entrevistas; 2) Grado de completitud y calidad en la realización de las encuestas y entrevistas con el uso de las TIC; y 3) Nivel de aceptación por parte de los entrevistados y encuestados sobre la calidad del instrumento realizado.

> Analizar de forma estadística los datos obtenidos en la utilización de métodos investigativos. Esta habilidad permitirá al investigador utilizar las TIC con el propósito de obtener información 
estadística a partir de los datos obtenidos. Sus indicadores pudieran ser: 1) Selección efectiva de las herramientas informáticas a utilizar para el análisis estadístico; 2) Grado de operación con la herramienta seleccionada; y 3) Nivel de interpretación de los resultados obtenidos.

$>$ Búsqueda, selección bibliográfica, y aplicación de normas bibliográficas con gestores bibliográficos. Esta habilidad le permitirá obtener a partir del empleo de base de datos académica e institucional, buscadores académicos Web, y buscadores temáticos, información asociada a posibles soluciones a las deficiencias, limitaciones y carencias que el investigador encontró en el diagnóstico realizado. Sus posibles indicadores son: 1) cantidad de artículos, libros, monografías, reflexiones, y demás documentos bibliográficos encontrados; y 2) utilización correcta de gestores bibliográficos en el momento de citar trabajos consultados.

$>$ Intercambiar en foros y comunidades científicias y comunidades colaborativas. Esta habilidad le permitirá al investigador intercambiar a través de las redes académicas en Internet, información con otros investigadores, obteniendo así información y contribuyendo a la socialización del conocimiento en áreas de interés común. Sus indicadores son: 1) Determinar a partir de una búsqueda en la red de redes, comunidades científicias, colaborativas, y en redes académicas, cuales le permitirán obtener información necesaria para su investigación; 2) nivel de interacción comunicativa a través de las TIC en dichas redes y comunidades; 3) uso eficiente de las potencialidades tecnológicas que los sitios web poseen, en las cuales se encuentran las redes y comunidades.

Utilizar eficientemente los editores de textos y otro software que permitan visualizar los resultados actuales de la investigación. Esta habilidad permite al investigador utilizar los editores de textos para la escritura de la memoria escrita de la investigación, en correspondencia del momento en que se encuentra la investigación. Sus indicadores son: 1) Uso correcto de las funcionalidades que posee el editor de texto (desempeño en el uso del editor de texto); y 2) Uso correcto de otro software que permitan al investigador la visualización de los resultados actuales de la investigación como por ejemplo el PowerPoint u otros similares.

En la etapa de "Planificación de la actividad científica" el investigador debe determinar: los componentes del diseño teórico-metodológico de la investigación a partir del problema formulado (objeto, objetivos, campo de acción, hipótesis (idea a defender o preguntas científicas), categorías y variables, resultados, tareas, población y muestra, métodos, técnicas y procedimientos); los plazos necesarios para la obtención de los resultados; y los recursos materiales y humanos necesarios para la obtención y la introducción de los resultados; además debe determinar anticipadamente las formas y vías para la introducción en la práctica de los resultados; diseñar Proyectos de Investigación-Desarrollo e Innovación Tecnológica (en dependencia del tipo de investigación y del alcance de esta); diseñar Proyectos de Tesis de Diploma, Maestría y Doctorado (en dependencia del tipo de investigación); diseñar Proyectos de Gestión de Recursos (en dependencia del tipo de investigación y del alcance de esta); y por último identificar posibles instituciones financieras de los Proyectos de Gestión (en dependencia del tipo de investigación y del alcance de esta).

En todas las etapas de la investigación se puede y debe hacerse uso de las TIC ya que propician en menor tiempo la obtención de resultados.

Las habilidades asociadas al uso de las TIC para la segunda estapa son:

Utilizar eficientemente los editores de textos y otro software que permitan visualizar los resultados actuales de la investigación. Se mantiene esta habilidad debido a la necesidad de interacción entre la investigación y los editores de textos y otro software. 
$>$ Utilizar de forma eficiente herramientas para confección de cronogramas de trabajos. Esta habilidad le permite al investigador confeccionar cronogramas de trabajo a partir de las potencialidades que ofrecen las TIC, como por ejemplo el Microsoft Project y el Gantt Project. En este sentido los estudiantes de carreras con perfil informático pueden integrar herramientas de gestión de proyectos informáticos. Los indicadores para esta habilidad son: 1) el nivel de uso de las potencialidades que brinda el software para la realización de cronogramas de trabajo; y 2) nivel de conocimiento que posea sobre la herramienta informática.

La tercera etapa de la investigación es la "Ejecución de la investigación" en la que el investigador debe ejecutar las tareas planificadas según cronograma; controlar la ejecución de las tareas; evaluar sistemáticamente los avances en el logro de los resultados y objetivos de la investigación; ejecutar el presupuesto asignado a la investigación; y controlar los recursos asignados de la investigación.

En esta etapa, al igual que en la primera y segunda, las habilidades asociadas al uso de las TIC que se mantienen con: Utilizar eficientemente los editores de textos y otros software que permitan visualizar los resultados actuales de la investigación; utilizar de forma eficiente herramientas para confección de cronogramas de trabajos ya que el investigador debe consultar el estado actual de su investigación en correspondencia con el cronograma y si es necesario modificarlo, se procede a realizarlo; y la aplicación de normas bibliográficas con gestores bibliográficos.

Otras habilidades que se pudieran tener en cuenta son:

$>$ Aplicar correctamente las TIC en el desarrollo de la investigación. Esta habilidad se encuentra en correspondencia a las características propias de la profesión que esté estudiando el universitario, como por ejemplo en las carreras técnicas las asociadas a la computación, la informática, la ingeniería eléctrica, ingeniería industrial y arquitectura; u otras profesiones como las carreras pedagógicas y las de ciencias sociales y humanísticas, donde el empleo de las TIC pueden variar. Sus indicadores son: 1) Calidad en el uso de las TIC en el cumplimiento de las tareas de la investigación; 2) nivel de conocimiento que posea sobre la herramienta, software, hardware, u otra aplicación utilizada sea informática o no; 3) Instalación, configuración y utilización correcta de las TIC empleadas; y 4) Integración efectiva entre varias plataformas, herramientas informáticas, hardware, dispositivos electrónicos, y demás tipos de TIC.

> Emplear medios de comunicación en el ámbito de las TIC. Esta habilidad le permite al investigador utilizar correctamente las TIC como vía de comunicación entre tutor-estudiante investigador, e investigador-otras personas que se encuentran involucradas en el proceso investigativo. Los indicadores son: 1) Grado de utilización de las TIC como vía de comunicación; 2) Nivel de calidad en el uso adecuado de las TIC en la comunicación existente entre tutor y el investigador, y demás implicados.

Utilizar correctamente las TIC como vía de almacenaje de la investigación. Esta habilidad le permite al investigador asegurar que el trabajo realizado hasta el momento ha quedado guardado y seguro ante incidentes y acceso indebidos. Sus indicadores son: 1) Grado de concientización de la protección de la información investigativa; 2) Utilización efectiva de las TIC para salvaguardar el trabajo investigativo obtenido hasta el momento; 3) Creación y empleo adecuado de una Base de Datos o un servidor en el cual se guarden automáticamente los cambios realizados en la investigación (documento escrito).

En la etapa de "Procesamiento de la información" el investigador debe procesar los datos empíricos (categorizar, codificar, tabular y ordenar en tablas y gráficos); valorar estadísticamente los datos; interpretar los datos en correspondencia con la teoría; producir nuevas contribuciones a la teoría o 
contribuciones prácticas (depende del tipo de investigación y su alcance); formular conclusiones; y formular recomendaciones.

En esta etapa se evidencian habilidades en el uso de las TIC ya abordadas anteriormente como: 1) Utilizar eficientemente los editores de textos y otros software que permitan visualizar los resultados actuales de la investigación; 2) utilizar de forma eficiente herramientas para confección de cronogramas de trabajos ya que el investigador debe consultar el estado actual de su investigación en correspondencia con el cronograma y si es necesario modificarlo, se procede a realizarlo; 3) elaborar encuestas y entrevistas con la utilización de las TIC; 4) analizar de forma estadística los datos obtenidos en la utilización de métodos investigativos; 5) Aplicación de normas bibliográficas con gestores bibliográficos; 6) emplear medios de comunicación en el ámbito de las TIC; y 7) utilizar correctamente las TIC como vía de almacenaje de la investigación.

Otra habilidad que puede evidenciarse en esta etapa en relación a las TIC es analizar los resultados matemáticos obtenidos con el empleo de las TIC. Esta habilidad le permite al investigador hacer uso de herramientas matemáticas que le permitan gestionar y obtener información a partir de los datos obtenidos en los resultados investigativos y expresarlos a partir de tablas comparativas y gráficos (no necesariamente estadísticos). Sus indicadores son: 1) Uso correcto de la herramienta matemática; y 2) Conocimientos que posee sobre las funcionalidades de la herramienta.

En la etapa de "comunicación de los resultados científico-técnicos" el investigador debe presentar por escrito los resultados conforme a las normas de la redacción científica; exponer oralmente los resultados conforme a las normas del discurso científico; e intercambiar criterios con otros/as colegas.

En esta etapa se evidencian habilidades en el uso de las TIC ya abordadas anteriormente como: 1) Utilizar eficientemente los editores de textos y otros software que permitan visualizar los resultados actuales de la investigación; 2) elaborar encuestas y entrevistas con la utilización de las TIC; 3) analizar de forma estadística los datos obtenidos en la utilización de métodos investigativos; 4) Aplicación de normas bibliográficas con gestores bibliográficos; 5) emplear medios de comunicación en el ámbito de las TIC; 6) utilizar correctamente las TIC como vía de almacenaje de la investigación; y 7) intercambiar en foros y comunidades científicias y comunidades colaborativas.

Otras de las habilidades que se pudieran estar desarrollando son la búsqueda y selección de revistas y eventos científicos en la red de redes. Esta habilidad permite al investigador hacer uso de buscadores y metabuscadores Web, así como de base de datos nacionales e internacionales de espacios científicos en los cuales poder comunicar los resultados, ya sea en revistas de investigación científica, de divulgación científica y cultural o técnico-profesional, así como eventos científicos que aborden el tema investigado, y redes académicas. Los indicadores son: 1) Nivel de selección adecuada de los medios en que va a comunicar los resultados a la comunidad científica nacional e internacional; 2) Empleo correcto de los buscadores y metabuscadores Web y base de datos nacionales e internacionales.

En la etapa de "introducción y generalización de los resultados científico-técnicos en la práctica" el investigador debe acreditar la propiedad intelectual de los resultados científico-técnicos; ejecutar las acciones planificadas para la introducción de los resultados científico-técnicos; tramitar las certificaciones de la introducción en la práctica de los resultados científico-técnicos; ejecutar el presupuesto asignado para la introducción de los resultados; controlar los recursos asignados para la introducción de los resultados; y asesorar a los/las directivos en el proceso de generalización de los resultados científico-técnicos. 
Las habilidades que se pueden desarrollar asociadas al uso de las TIC en esta etapa son (depende del tipo de investigación y las condiciones tecnológicas que se necesiten para su introducción y generalización):

- Utilizar correctamente las TIC en la formación y capacitación de otras personas que van a utilizar los resultados obtenidos en la investigación. Esta habilidad le permite al investigador utilizar correctamente las TIC en las capacitaciones a realizar. Sus indicadores son: 1) Selección adecuada de las TIC que se vayan a utilizar en la capacitación, según las condiciones económicas, y tecnológicas existentes; y 2) instalación, estructuración, configuración y empleo adecuado de las TIC en la capacitación a realizar.

\section{Discusión}

Enmarcar el empleo de las TIC en la investigación científica a solo al uso de los gestores bibliográficos, la utilización de los buscadores temáticos, la presentación de los resultados obtenidos en la investigación; la comunicación interpersonal y trabajo colaborativo en redes; el procesamiento de textos; el uso de base de datos; la utilización de la hoja de cálculo; el uso de instrumentos de recogida de información; y el uso de herramientas para el análisis de datos; sería limitar las potencialidades que ofrecen las TIC al investigador.

Todas las habilidades que se proponen se encuentran en correspondencia con las condiciones tecnológicas que posea el investigador de forma personal e institucional; además del conocimiento y habilidades que posea en el uso de las TIC.

La mayoría de las habilidades investigativas asociadas al empleo de las TIC que se proponen pueden ser desarrolladas en todas las carreras universitarias pero en el caso de estudiantes de carreras con perfil informático se hace necesario tener en cuenta (entre otras),

1. Diseño e implementación de base de datos o servidores para salvaguardar la información de la investigación.

2. Determinación coherente de las herramientas tecnológicas y tecnologías de software y hardware a emplear en la investigación.

3. Determinación de la repercusión social de diseño, desarrollo, despliegue y mantenimiento de un sistema informático realizado desde una perspectiva científica e investigativa.

4. En el despliegue de un sistema informático, determinación de las herramientas y tecnologías necesarias para la realización de una capacitación ya sea a distancia o presencial. Si bien generalmente los estudiantes de esta carrera o similares, no logran desplegar el sistema informático que realizan como tesis de diploma, sería apropiado establecer estrategias didácticas que permitan desarrollar estas habilidades en vista al desempeño del estudiante en el ámbito laboral.

\section{Conclusiones}

La sistematización teórica realizada permitió constatar el poco uso que los autores le confieren a las TIC en la formación y desarrollo de las habilidades investigativas, enmarcándola solamente en algunos momentos del proceso del desempeño del investigador.

Las habilidades investigativas asociadas al uso de las TIC, no solo puede concebirse en determinados períodos de la actividad investigativa, sino que puede evidenciarse en cada una de las etapas o fases de la investigación científica, identificándose para ello habilidades específicas por cada una de ellas. 
Se hace necesario potenciar el desarrollo de estas habilidades en el contexto del perfil profesional de las carreras universitarias. En el caso de las carreras con perfil informático, se pueden vincular a las exigencias del desarrollo de software (industrial o no) desde una perspectiva investigativa y científica.

\section{Referencias bibliográficas}

1. Arbeláez, R., Orlando, N., y Ilce, M. (2006). El desarrollo de la competencia investigadora en los estudiantes de pregrado. Docencia Universitaria, 7(1), 5-10.

2. Association pour l'emploi des cadres (France), Deloitte (France). (2010). Skills and competencies needed in the research field objectives 2020. Paris: APEC.

3. Balbo, J. (2008). Formación en competencias investigativas, un nuevo reto en las universidad. Caracas: Universidad Central de Venezuela.

4. Baltrūna, A. (2013). (2013). The Essence of Learning Methodological Model and its Application in Developing TourismStudents' Research Competence. The Macrotheme Review, 2(1), 21-25.

5. Barnett, B. (2008). Using action-research strategies and cohort structures to ensure research competence for practitioner-scholar leaders. Journal of Research on Leadership Education, $3(1), 10-14$.

6. Bezrukova, N., y Bezrukov, A. (2013). Methodological basis of development of research competence of secondary school students in the network research community. Prague-Czech Republic: CBU international conference on integration and innovation in science and education.

7. Bolgzda, I., y Olehnovica, E. (2012). Structure of Doctoral Students' Research Competences in Sustainability Context. World Academy of Science. Engineering and Technology(66), 1.-3.

8. Bosco, A. (2005). Las TIC en los procesos de convergencia europea y la innovación en la universidad: oportunidades y limitaciones. Aula Abierta, 3-27.

9. British Educational Communications and Technology Agency (BECTA). (2007). Harnessing Technology Review 2007: Progress and impact of technology in education. United Kingdom: Department for Education of England.

10. Burke, L., Schlenk, E., Sereika, S., Cohen, S., Happ, M. B., y Dorman, J. (2005). Developing Research Competence to Support Evidence-Based Practice. Journal of Professional Nursing, 21(6), 358-363.

11. Castillo, S. (2008). Competencias investigativas desarrolladas por docentes de Matemática. ACTA SCIENTIAE. Revista de Ensino de Ciências e Matemática, 10(2), 3-7.

12. Castillo, S. (2011). Evaluación de competencias investigativas. Recife-Brasil: XII Conferencia Interamericana de Educación Matemática (CIAM 2011).

13. Chu, S., Chow, K., Tse, S., y Kuhlthau, C. C. (2008). Grade 4 Students' Development of Research Skills Through Inquiry-Based Learning Projects. School Libraries Worldwide, 10-37.

14. Contreras, Z. D. (2010). Las competencias investigativas. Educando. Ministerio de Educación de la República Dominicana.

15. Delamare Le Deist, F., y Winterton, J. (2005). What Is Competence? Human Resource Development International, 8(1), 27-46.

16. European Commision. (2006). Benchmarking Access and Use of ICT in European Schools 2006. Final Report from Head Teacher and Classroom Teacher Surveys in 27 European Countries. Bonn-Germany: Empirica.

17. Estrada, O. (mayo-agosto, 2014). Sistematización teórica sobre la competencia investigativa. Revista Electrónica Educare, 18(2), 177-194

18. Facundo, L., Patricia, D., Carlos, J., Ruíz, Y., Claro, A., y Vanegas, H. (2007). Visibilidad y formación en investigación. Estrategias para el desarrollo de competencias investigativas. STUDIOSITAS, 2(2), 10-12. 
19. Gayol, M. D., Montenegro, S. M., Tarrés, M. C., y D’Ottavio, A. E. (2008). Competencias Investigativas. Su desarrollo en carreras del Área de la Salud. UNI-PLURI/VERSIDAD, 8(8), 58.

20. Hsing-fen, L., Miozzoa, M., y Laredob, P. P. (2010). Career patterns and competences of PhDs in science and engineering in the knowledge economy: The case of graduates from a UK research-based university. Research Policy (39), 869-878.

21. Irigoyen, J. J., Yerith, M., y Karla, F. (2011). Competencias y educación superior. Revista Mexicana de Investigación Educativa, 16(48), 244.

22. Maldonado, L. F., Landazábal, D. P., y Hernández, J. C. (2007). Visibilidad y formación en investigación. Estrategias para el desarrollo de competencias investigativas. Revista Studiositas, 2(2), 43-56.

23. Muñoz, J., Quintero, J., y Munévar, R. (2006). Cómo desarrollar competencias investigativas en Educación. Bogotá: Magisterio.

24. Ortega, E., y Jaik, A. (2010). Escala de evaluación de competencias investigativas. Praxis Investigativa REDIE, 2(3), 72-80.

25. Pla, R. (2005). Las competencias profesionales para el desempeño del docente en la educación de los alumnos desde un enfoque integrador. La Habana: Congreso Internacional Pedagogía 2005.

26. Quigley, B., y Kuhne, G. (1996). Creating practical knowledge through action research: Posing problems, solving problems, and improving daily practice. New Directions for Adult and Continuing Education(73), 7-10.

27. Rodríguez, M. (2000). Formación reflexiva-creativa de competencias investigativas en los docentes. Buenos Aires: CLACSO.

28. Tobón, S. (2008). La formación basada en competencias de la Educación Superior. El enfoque complejo. Guadalajara-México: Universidad autónoma de Guadalajara.

29. Tobón, S. (2008a). La formación basada en competencias en la Educación Superior: el enfoque complejo. Bogotá: Instituto Cife.ws.

30. Tobón, S., Rial, A., Ángel, M., y García, J. A. (2006). Competencias, calidad y educación superior (Primera Edición ed.). Bogotá: Magisterio.

31. Velásquez, R. (2007). De estoy aquello sobre las habilidades investigativas. Avances Investigación en Ingenierías(7), 4-7. 